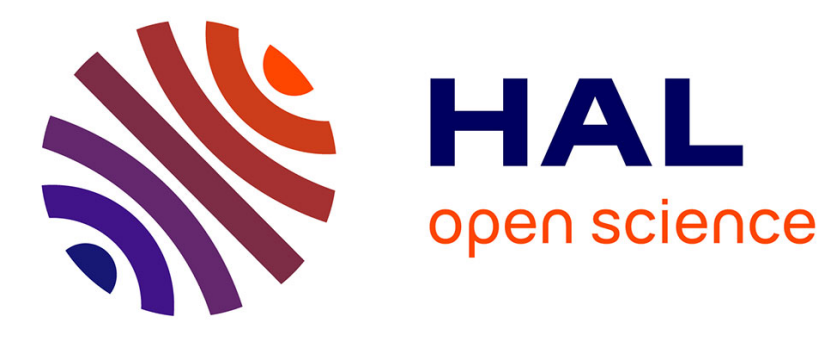

\title{
La recherche documentaire informatisée à l'école
} Jérôme Dinet, Jean-Michel Passerault, Jean-François Rouet

\section{To cite this version:}

Jérôme Dinet, Jean-Michel Passerault, Jean-François Rouet. La recherche documentaire informatisée à l'école: Vers une modélisation des processus cognitifs liés au jugement de pertinence des références documentaires chez les élèves de CM2. Cinquième colloque Hypermédias et apprentissages, Apr 2001, Grenoble, France. pp.135-150. edutice-00000457

\section{HAL Id: edutice-00000457 https://edutice.archives-ouvertes.fr/edutice-00000457}

Submitted on 8 Jun 2004

HAL is a multi-disciplinary open access archive for the deposit and dissemination of scientific research documents, whether they are published or not. The documents may come from teaching and research institutions in France or abroad, or from public or private research centers.
L'archive ouverte pluridisciplinaire HAL, est destinée au dépôt et à la diffusion de documents scientifiques de niveau recherche, publiés ou non, émanant des établissements d'enseignement et de recherche français ou étrangers, des laboratoires publics ou privés. 


\title{
LA RECHERCHE DOCUMENTAIRE INFORMATISÉE À L'ÉCOLE
}

\section{Vers une modélisation des processus cognitifs liés au jugement de pertinence des références documentaires chez les élèves de CM2}

\author{
Jérôme DINET****, Jean-Michel PASSERAULT* \\ et Jean-François ROUET* \\ * Laboratoire Langage et Cognition (LaCo-CNRS UMR 6096) - MSHS \\ 99, avenue du recteur Pineau - BP 632 - 86022 Poitiers Cedex \\ ** CRDP de Poitou-Charentes - 6, rue Sainte-Catherine - 86034 Poitiers Cedex \\ jerome.dinet@mshs.univ-poitiers.fr, \\ jean-michel.passerault@mshs.univ-poitiers.fr, \\ jean-francois.rouet@mshs.univ-poitiers.fr
}

\begin{abstract}
Résumé : Les volontés institutionnelles encouragent l'utilisation de l'ordinateur pour les recherches d'informations. Dans cette étude, nous nous intéressons à la sélection de références documentaires pertinentes en nous focalisant sur les impacts de trois caractéristiques des listes de références sur le jugement de pertinence de 34 élèves de CM2 : la familiarité thématique, la longueur de la liste de références documentaires et la pertinence de ces références. Les résultats ont montré que ces trois facteurs ont des influences significatives sur le nombre de références pertinentes sélectionnées, sur le temps nécessaire à cette sélection et plusieurs interactions ont été trouvées. Nous avons utilisé un modèle récent de compréhension et de mémorisation pour conceptualiser les processus cognitifs impliqués dans cette tâche de sélection. Les implications pour l'éducation et la conception d'outils électroniques sont discutées.
\end{abstract}

Mots-clés : recherche documentaire informatisée, activation conceptuelle, jugement de pertinence, école primaire.

Abstract: Educational policies encourage the use of computers to search information. In the present study, we concentrated on the selection of relevant book references and particularly we focused on the impact of three characteristics of the lists of references on the judgement of relevance by 34 pupils in Grade 5: the topic's familiarity, the length of book reference list and the relevance of these book references. Results showed that the three factors significantly influenced the number of relevant references selected and the time which was necessary to perform this selection, moreover several interactions were found. We used a recent model of text 
comprehension and memorisation to conceptualise the cognitive processes involved in this task of selection. Implications for education and for the conception of electronic tools are discussed.

Keywords : computerised information search, conceptual activation, judgement of relevance, primary grade.

\section{INTRODUCTION}

Aujourd'hui, face à l'augmentation et la diversification de la masse documentaire pour l'éducation, les élèves ne peuvent plus se contenter de rechercher des informations, documents et références documentaires de manière manuelle, en «butinant » dans les fonds de leurs écoles. De plus, les élèves ont désormais accès à des sources documentaires diverses et parfois très distantes physiquement. Ces modifications dans les sources documentaires disponibles pour les élèves et leurs accès ont rendu nécessaire le recours à des outils informatiques de recherche documentaire. Quels que soient l'outil et le contexte de réalisation, l'activité qui consiste à localiser dans des bases de données électroniques des références de documents pertinents à l'aide d'un outil informatique correspond à la recherche documentaire informatisée, généralement notée RDI (Barès, 1984 ; Sutter, 1984). identifiables :

Généralement, une RDI requiert a minima deux étapes clairement

- l'étape d'interrogation documentaire: après avoir traité les consignes et l'énoncé thématique, l'utilisateur (ici, l'élève) doit produire des termes de recherche. C'est sur la base de ces termes de recherche, aussi appelés requêtes, que l'outil effectue sa recherche dans une base de données définie ;

- l'étape de sélection : pour une requête documentaire donnée, l'outil répond habituellement en proposant un ensemble de résultats potentiellement pertinents. L'élève doit alors opérer une sélection des références pertinentes parmi l'ensemble proposé. Cette étape de sélection des références pertinentes est cruciale puisque l'atteinte de l'objectif initialement prévu (rédiger un article, préparer une exposition, etc.) peut en dépendre. Mais, quels sont les processus cognitifs sous-jacents à cette activité ?

À l'aide d'une expérience reportée ici et d'un modèle récent de psychologie cognitive (van den Broek, Fletcher \& Risden, 1993; van den Broek, Risden \& Husebye-Hartmann, 1995 ; van den Broek et al., 1996 ; van den Broek et al., 1999), nous tentons d'appréhender et de modéliser les processus cognitifs qui interviennent dans la sélection de références documentaires chez des élèves de CM2.

\section{L'importance des mots clés dans la sélection de références documentaires}

Si chez l'adulte c'est le sens global de la référence documentaire qui constitue le principal facteur de choix, des indices purement perceptifs (tels que le marquage typographique) sont susceptibles d'influencer le jugement de pertinence de références documentaires chez des élèves de CM1 et de CM2 (Dinet, Rouet \& 
Passerault, 1998). L'impact de ce facteur perceptif «attrayant» est également retrouvé par Hirsh (2000) qui a identifié neuf critères pouvant influencer la sélection de références, de documents ou d'informations chez des élèves de Grade 5. Parmi ces 9 critères apparaît la pertinence par rapport au thème. Même si la distinction entre une référence pertinente et une référence non pertinente est complexe à définir avec précision (pour une synthèse récente, voir Mizzaro, 1998), sont considérées comme pertinentes les références qui permettent de localiser et donc de traiter ultérieurement des documents en vue d'atteindre un objectif plus ou moins bien défini (rédiger un article scientifique, préparer un colloque, ses vacances, apprendre des informations, etc.) relativement à un thème prédéfini (Lefort, 1990). Or, comme le montrent de nombreuses études menées chez l'adulte et chez l'enfant (Borgman et al., 1990 ; Kuhlthau, 1991; Marchionini, 1995 ; Moore \& St.George, 1991 ; Osmont, 1995 ; Solomon, 1993), l'identification des mots-clés dans les références est fondamentale dans ce processus de jugement de pertinence des références documentaires puisque ce n'est qu'en les identifiant que l'individu peut évaluer la pertinence des références.

\section{Activation conceptuelle et sélection de références documentaires}

D'un point de vue psychologique, le traitement de l'énoncé thématique et de la question initiale entraînent un premier processus de diffusion de l'activation dont le but est la construction d'une représentation intégrée et cohérente du sens de la question et des consignes (Kintsch, 1998 ; Kintsch \& van Dijk, 1978 ; van den Broek, Risden \& Husebye-Hartmann, 1995). Ainsi, selon le stock de connaissances référentielles dont dispose l'individu, le thème " la pollution des eaux » peut activer par le biais d'inférences un vecteur de termes associés comme par exemple " poissons », " marée noire », " écologie », « écosystème », « phosphates », 《 nitrates », etc. Le vecteur exact d'activation, c'est-à-dire la liste des concepts activés par ce processus de propagation de l'activation ainsi que le niveau d'activation qu'ils atteignent dépendent d'un large ensemble de facteurs, au premier plan desquels figurent les connaissances initiales propres à chaque individu, c'est-à-dire le niveau de familiarité du thème de la RDI. Van den Broek et ses collaborateurs (van den Broek et al., 1993, 1995, 1996, 1999) proposent un modèle de compréhension et de mémorisation de textes dans lequel cette configuration de concepts actifs est appelée « cohorte ». Selon ces auteurs, le contenu de la cohorte n'est pas figé, mais se modifie dynamiquement au fil de l'activité, à mesure des informations rencontrées dans le texte.

Si l'on considère que la cohorte initiale comprend tous les concepts activés après le traitement des consignes et de la question, que devient cette cohorte dans le cas où l'individu, de surcroît un jeune élève, est confronté à des listes de références, celles-ci étant tantôt adéquates tantôt inadéquates par rapport au thème ? On peut faire l'hypothèse qu'elle est soumise à deux tendances contradictoires : oubli et maintien. En accord avec le modèle de van den Broek, la lecture de références successives entraîne une modification de la cohorte puisque chaque nouveau concept activé "chasse » les précédents faute de place en mémoire de travail, sauf si ces concepts déjà présents sont de nouveau explicitement mentionnés ou fortement associés aux concepts entrants (van den Broek et al., 1996). Si l'application stricte de ce modèle permet de simuler correctement la compréhension de textes narratifs, 
elle conduit à un résultat apparemment paradoxal dans le cas de la RDI : en effet, s'il n'est pas périodiquement répété, l'objectif de recherche (le vecteur initial) est condamné à la désactivation progressive à mesure que le sujet rencontre des références comportant des termes différents, surtout si ces termes ne sont pas reliés au thème de la RDI. En d'autres termes, on oublierait donc ce que l'on cherche sauf à le trouver tout de suite! L'examen de données empiriques (Brorowczak \& Machut, 1997 ; Chollet, 1997 ; Osmont, 1995 ; Solomon, 1993) montre que l'oubli de la question initiale constitue effectivement l'un des problèmes les plus fréquents lors de la recherche d'informations chez l'adulte non expert en RDI comme chez l'enfant. Ce phénomène pourrait donc refléter la compétition entre les concepts initiaux de la cohorte et les concepts « entrants » à chaque nouveau cycle de lecturecompréhension des références documentaires successives.

L'oubli de la question n'est toutefois pas systématique, et il faut donc imaginer que la "cohorte » initiale bénéficie d'un statut spécial en mémoire qui lui permet de se maintenir à mesure que le sujet inspecte une liste de références. Ce maintien est par ailleurs indispensable à l'opération d'évaluation (Hirsh, 2000 ; Rouet \& Tricot, 1998), qui conduit à la décision de retenir ou de rejeter une information (ici, une référence documentaire). Or, le sujet ne peut décider si une référence est pertinente ou pas qu'après l'avoir comparée à sa représentation de l'objectif, qu'il doit pour cela avoir activement à l'esprit.

Quoi qu'il en soit, sur la base du modèle de van den Broek et al. (1995, 1996, 1999), nous pouvons supposer que, à défaut de réactivation volontaire, la représentation qu'a un élève du thème de la RDI risque de se dégrader peu à peu, à mesure que ce même élève est obligé de traiter des références documentaires. En effet, la cohorte conceptuelle activée lors du traitement des consignes et du thème se dégrade progressivement au fil de l'activité de lecture-compréhension des références documentaires, entraînant ainsi des difficultés de jugement de pertinence de ces mêmes références. Cette dégradation de la représentation doit conduire à des difficultés de jugement de pertinence des références documentaires. Nous pouvons également supposer que certains facteurs peuvent accélérer cette dégradation et donc encore plus perturber le jugement de pertinence des élèves.

\section{Opérationnalisation et hypothèses}

Lors d'une tâche de sélection de références documentaires pertinentes parmi un ensemble de références, sur la base du modèle de van den Broek et al. (1995, 1996, 1999), nous pouvons supposer que trois facteurs essentiels sont susceptibles d'avoir des impacts sur la dégradation du vecteur initial :

- le niveau de familiarité du thème de la RDI : ce facteur doit influencer les capacités de jugement de pertinence dans le sens où la dégradation de la cohorte initiale doit être plus rapide dans le cas où les concepts constitutifs de la cohorte conceptuelle initiale sont peu nombreux, c'est-à-dire dans le cas où le thème est peu familier. Ainsi, le jugement de pertinence doit être plus difficile à réaliser lorsque le thème de la RDI est peu familier pour l'élève ;

- la longueur des listes de références : ce facteur doit également jouer un rôle dans le sens où la cohorte conceptuelle initiale disparaît progressivement au fil de la lecture-compréhension d'un ensemble important de références 
documentaires. Le jugement de pertinence de références documentaires doit donc être plus difficile à réaliser pour un jeune élève à la fin d'une liste qu'au début de la même liste ;

- l'adéquation des références par rapport au thème: dans une liste de références documentaires, la pertinence des premières doit influencer le jugement porté sur des références suivantes. En effet, le traitement de références non pertinentes et donc l'entrée de nouveaux concepts non pertinents en mémoire doit provoquer et accélérer «l'effacement» de la cohorte initiale à cause des capacités limitées de la mémoire de travail. Au contraire, si la liste est uniquement composée de références adéquates, on peut penser que leur traitement permettra de maintenir active la cohorte initiale, par renouvellement ou propagation des activations initiales (van den Broek \& al., 1995, 1996, 1999).

Dans l'expérience présentée ici, nous nous sommes intéressés à l'impact des trois facteurs explicités ci-dessus sur la sélection de références documentaires pertinentes par des élèves de CM2. Ces élèves avaient reçu pour consigne de sélectionner des références documentaires pertinentes parmi un ensemble de références documentaires. Plusieurs thèmes, prédéfinis par les expérimentateurs, ont été utilisés : la moitié de ces thèmes étaient familiers et l'autre étaient très peu familiers pour les élèves. À chacun des thèmes correspondait une liste de références. Dans chacune de ces listes, les expérimentateurs avaient placé une référence documentaire adéquate par rapport au thème correspondant. Cette référence était appelée « référence cible ». Pour chacun des thèmes (familier versus non familier), la cible pouvait être placée après une ou trois autres références documentaires, c'està-dire en position 2 ou en position 4, afin de tester l'impact de la longueur de la liste. Enfin, les références situées avant la cible pouvaient être adéquates ou non par rapport au thème. Dans notre expérience, nous nous sommes uniquement intéressés au jugement de pertinence porté par les élèves sur cette référence cible.

Deux indicateurs ont été utilisés pour rendre compte de ce jugement de pertinence :

- le nombre de références cibles sélectionnées par les élèves (et donc évaluées adéquates par ces élèves) ;

- le temps nécessaire aux élèves pour effectuer cette sélection. Cet indicateur temporel représente un indice capable de rendre compte du niveau d'activation des concepts constitutifs de la cohorte initiale tout au long de l'activité. Si le niveau d'activation de la cohorte initiale diminue, alors le temps nécessaire à la sélection des références cibles (qui contiennent des concepts reliés à cette cohorte initiale) doit augmenter.

Pour résumer, nous avons testé les hypothèses suivantes :

- le nombre de références cibles (donc pertinentes) sélectionnées par les élèves doit diminuer lorsque :

- les références cibles sont placées après trois autres références documentaires (hypothèse 1), 
- les références documentaires qui précèdent les cibles sont inadéquates par rapport au thème (hypothèse 2),

- le thème de la RDI est peu familier pour les élèves (hypothèse 3) ;

- le temps nécessaire à un élève pour sélectionner et donc évaluer comme pertinentes les références cibles doit augmenter lorsque :

- les références cibles sont placées après trois autres références documentaires (hypothèse 4),

- les références documentaires qui précèdent les cibles sont inadéquates par rapport au thème (hypothèse 5 ),

- le thème de la RDI est peu familier pour les élèves (hypothèse 6).

En exposant ainsi nos hypothèses opérationnelles, nous supposons donc que le nombre de références cibles sélectionnées diminue et que le temps nécessaire à cette sélection augmente dans les mêmes conditions. De plus, les effets d'interaction sont également attendus puisque la représentation qu'a un élève du thème de la RDI risque de se dégrader progressivement à mesure que ce même élève traite des références documentaires surtout si celles-ci sont nombreuses, inadéquates et que l'élève possède peu de connaissances initiales sur le thème de la RDI.

\section{EXPÉRIENCE}

\section{Participants}

Pour participer à cette expérience, soixante-dix élèves de CM2 ont été recrutés dans deux groupes scolaires situés en zone périurbaine de la ville de Poitiers (académie de Poitiers). Tous les élèves étaient francophones. Tous pratiquaient des recherches documentaires informatisées (RDI) toutes les semaines, depuis au moins deux ans. Tous les élèves utilisaient le même logiciel de RDI que celui utilisé dans notre expérience pour obtenir des références documentaires. Tous étaient donc familiarisés avec l'affichage des références documentaires utilisées dans notre expérience.

Afin d'évaluer les possibles corrélations entre les capacités de lecturecompréhension, l'empan mnésique et l'activité de sélection de références documentaires, les 70 élèves devaient passer un test de lecture-compréhension (Lefavrais, 1989) et un test d'empan mnésique non verbal (Case, Kurland \& Goldberg, 1982 ; Siegel \& Ryan, 1989) avant l'expérience. Or, les analyses étant encore en cours lors de la soumission de cette communication, seuls les résultats de trente-quatre élèves qui ont été évalués «bons » ou «très bons » lecteurs-compreneurs et considérés comme ayant un fort empan mnésique sont présentés ici. En d'autres termes, dans cette communication, ce sont les résultats d'un sous-échantillon constitué d'élèves sans difficultés apparentes en lecture-compréhension et en mémorisation qui sont présentés. 


\section{Matériel}

\section{Niveau de familiarité des thèmes}

Dix-huit thèmes travaillés lors de RDI par les élèves de Cours Moyen deuxième année (CM2) de l'Académie de Poitiers (France) ont été retenus. Chacun de ces 18 thèmes était composé de deux concepts (par exemple, « la pollution des eaux'). Sur ces dix-huit thèmes, 12 ont été conservés après avoir demandé aux élèves de noter, lors de productions libres, tout ce qu'ils savaient sur chaque thème. Sur la base du nombre d'informations correctes produites sur tous les thèmes, ont pu être distingués :

- les 6 thèmes les plus familiers : la planète Terre / la pollution des eaux / les instruments de musique / les animaux sauvages / les sports de montagne / les poissons des rivières ;

- les 6 thèmes les moins familiers : les monnaies en Europe / l'histoire de l'Amérique / les montagnes d'Asie / l'invention de l'écriture / la période préhistorique / l'océan indien.

\section{Constitution des listes des références documentaires}

Pour chacun des 12 thèmes retenus ( 6 familiers et 6 non familiers), quinze références documentaires ont été extraites de la base documentaire initiale fournie avec le logiciel BCDI 2 école qui équipe la majorité des écoles primaires françaises (CRDP de Poitou-Charentes, versions 1.02 du 3 janvier 2000). Chaque référence contenait au moins l'un des deux concepts présents dans l'intitulé du thème de la RDI (par exemple, « planète » ou « Terre » pour le thème « la planète Terre »). Pour chaque thème, quatre élèves de CM2 ont sélectionné les quatre références les plus adéquates par rapport au thème (c'est-à-dire les plus intéressantes par rapport au thème) et les trois références les moins adéquates parmi les 15 références extraites de la base initiale. L'une des quatre références jugées adéquates était considérée comme « référence cible ». Selon les conditions expérimentales, cette référence cible était placée après une ou après trois références documentaires : cette cible pouvait donc occuper la seconde ou la quatrième position dans une liste. De plus, la ou les références documentaires précédant cette référence cible étai(en)t soit adéquate(s) soit inadéquate(s) par rapport au thème. Chaque élève voyait toutes les conditions pour les 6 thèmes familiers et les 6 thèmes non familiers.

La présentation et la nature des références documentaires étaient identiques à celles que les élèves manipulaient habituellement dans leurs écoles (figure 1). Le contenu de chaque référence documentaire était essentiellement textuel et comprenait : le niveau scolaire (exprimé en âge) auquel était destiné le document correspondant, le titre du document, le titre du périodique d'où était extrait le document s'il s'agissait d'un article, la nature du document (roman, documentaire, etc.), le numéro des pages correspondant à la séquence s'il s'agissait d'un article, un résumé, les mots-clés servant à l'indexation, la nature du support (périodique, livre, etc.), le nom de la collection s'il y avait lieu, la date d'exemplarisation, le nombre d'exemplaires présents dans le fonds et le nombre d'exemplaires disponibles. 


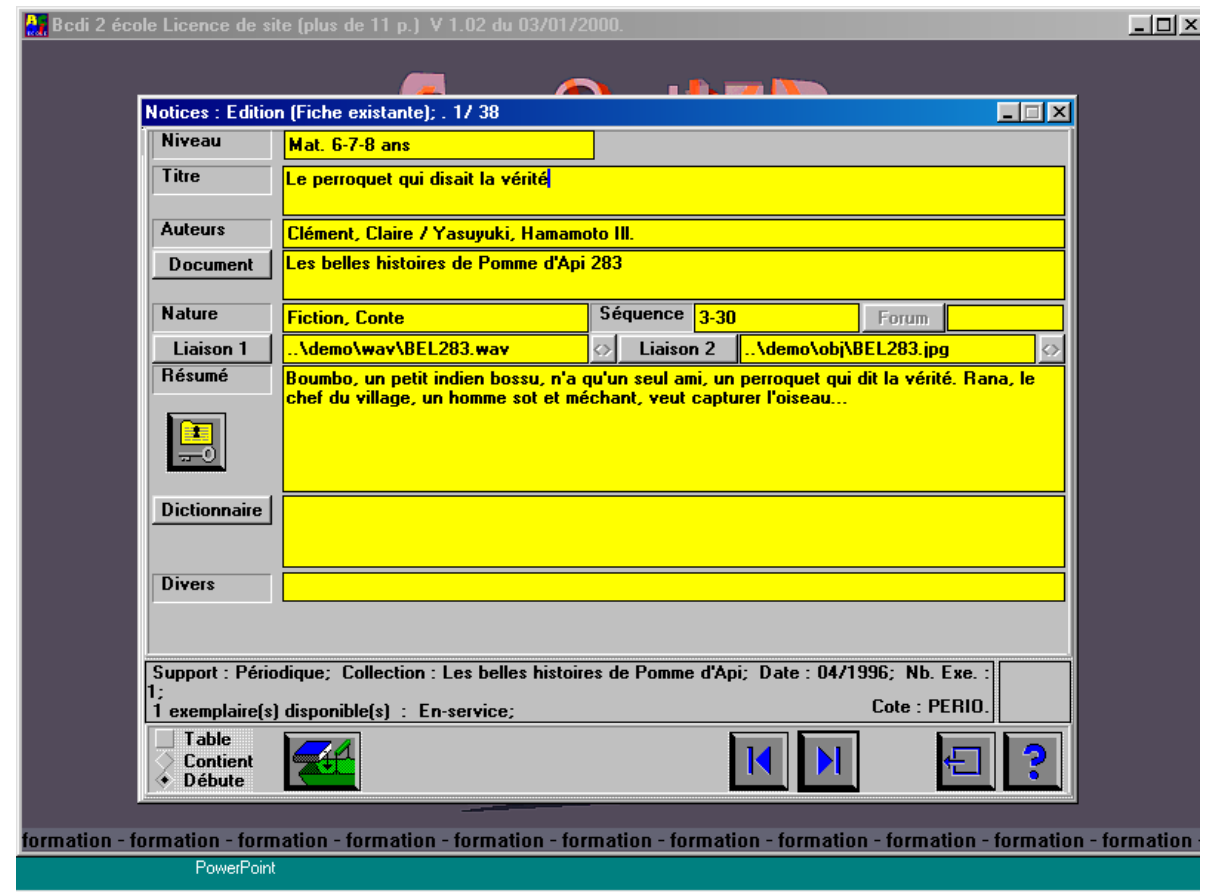

Figure 1. Exemple d'une référence documentaire extraite de la base de démonstration fournie avec le logiciel BCDI 2 école.

\section{Procédure}

La procédure comportait trois phases :

1. Deux semaines avant l'épreuve de sélection des références documentaires, le niveau de lecture-compréhension et l'empan de la mémoire de travail de chaque élève ont été évalués, respectivement avec le test dit de « la pipe et du rat » (Lefavrais, 1989) et un test d'empan mnésique non verbal (Case, Kurland \& Goldberg, 1982; Siegel \& Ryan, 1989). Dans cette communication, seuls les résultats d'une sous-population, à savoir 34 élèves ne présentant aucune difficulté ni en lecture-compréhension ni en ce qui concerne la mémoire de travail sont présentés.

2. Une semaine avant l'épreuve de sélection des références documentaires, chaque élève devait produire tout ce qu'il savait relativement aux dix-huit thèmes retenus par les expérimentateurs sur la base des thèmes travaillés dans différentes écoles de l'Académie de Poitiers. Cette production libre d'informations a permis de distinguer les 6 thèmes les plus familiers et les 6 thèmes les moins familiers pour les élèves.

3. Lors de l'épreuve de sélection de références documentaires, la passation était individuelle. Chaque élève était prévenu qu'il allait devoir sélectionner grâce à l'ordinateur les références documentaires les plus intéressantes («qui peuvent apprendre des choses ») sur douze thèmes différents. Dès que l'élève 
déclarait être prêt, il appuyait sur la barre «espace» du clavier de l'ordinateur et un thème était affiché à l'écran. Ce thème était précédé de la consigne suivante : «mets dans le panier les références les plus intéressantes, qui peuvent apprendre des choses sur [intitulé du thème]. » Dès qu'il le souhaitait, l'élève cliquait avec la souris et une référence documentaire apparaissait aussitôt. Deux options s'offraient à l'élève :

- si l'élève estimait cette référence adéquate par rapport au thème, il devait la mettre de côté en cliquant sur le bouton «mettre dans le panier » comme il avait l'habitude de le faire avec ce logiciel. Ensuite, il devait cliquer sur le bouton « suivante » pour faire apparaître une nouvelle référence ;

- si l'élève estimait que la référence était inadéquate, il devait simplement cliquer sur « suivante ».

Dès que l'élève parvenait à la fin d'une liste de références documentaires et qu'il cliquait sur « suivante », un nouveau thème apparaissait. Afin de s'assurer que la procédure était maîtrisée, quatre cycles d'essais ont été proposés à chaque élève avant le début de l'épreuve. L'ordre des thèmes et des listes de références était contrebalancé. Dans notre expérience, nous nous intéressons uniquement à la sélection des références cibles et au temps nécessaire à cette sélection.

\section{RÉSULTATS}

Les analyses statistiques ont été réalisées sur la base du plan suivant : $\mathrm{S} 34 *$ Familiarité thématique2 * Adéquation $2 *$ Position2

Avec trois facteurs intra-groupes :

- familiarité thématique à deux modalités, familier («Familier ») versus non familier («Non familier »);

- adéquation par rapport au thème des références qui précèdent la cible à deux modalités, adéquates ( Adéquate ») versus inadéquates (" Inadéquate ») ;

- position de la référence cible à deux modalités, seconde position («2») versus quatrième position (« $4 »)$.

\section{Le nombre de références cibles sélectionnées}

Pour chaque condition, nous avons calculé le nombre de références cibles « mises dans le panier» (figure 2). En d'autres termes, nous nous sommes intéressés au nombre de références cibles évaluées comme adéquates par les élèves sachant que toutes ces références cibles sont effectivement adéquates par rapport à leurs thèmes respectifs. 


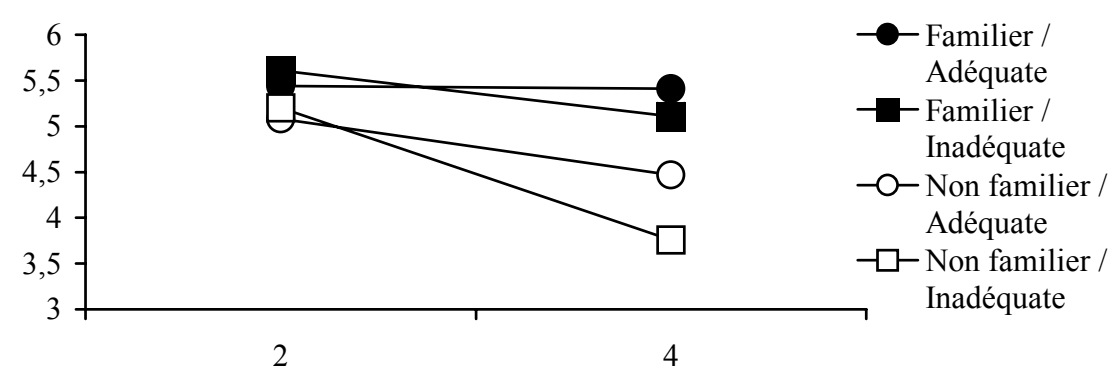

Position de la référence cible

\section{Figure 2. Nombre moyen de références documentaires cibles sélectionnées $(\min .=0 ; \max .=6)$.}

Des analyses de variance multiples montrent que l'effet d'interaction entre le niveau de familiarité thématique, la position de la référence cible et l'adéquation des références précédentes est significative $\left(F_{(1-33)}=18,15, p<.01\right)$. Les résultats montrent également que les élèves commettent plus d'erreurs de sélection lorsque le thème de la RDI leur est peu familier $\left(F_{(1-33)}=50,98, p<.01\right)$, que la référence documentaire cible soit précédée d'une ou de trois autres références documentaires (respectivement, $F_{(1-33)}=15,57, p<.01$ et $F_{(1-33)}=45,05, p<.01$ ), que ces références précédentes soient adéquates ou non (respectivement, $F_{(1-33)}=28,38, p<.01$ et $\left.F_{(1-33)}=30,09, p<.01\right)$.

De plus, les élèves sélectionnent moins souvent une référence documentaire cible si celle-ci est précédée de trois autres références documentaires $\left(F_{(1-33)}=39,09\right.$, $p<.01)$, surtout si ces références précédentes sont inadéquates par rapport au thème $\left(F_{(1-33)}=24,17, p<.01\right)$ et que le thème de la RDI est peu familier pour les élèves $\left(F_{(1-33)}=41,76, p<.01\right)$.

Par contre, l'adéquation des références qui précèdent la référence cible n'a pas d'effet significatif sur le nombre de sondes sélectionnées, sauf dans le cas où il y a en trois $\left(F_{(1-33)}=8,50, p<.01\right)$.

En d'autres termes, bien qu'elles soient pertinentes, les références documentaires cibles sont moins souvent sélectionnées si elles sont précédées de plusieurs autres références documentaires, surtout lorsque le thème de la RDI est peu familier pour les élèves. Ce phénomène est accentué lorsqu'il y a trois références inadéquates qui précèdent la référence cible, c'est-à-dire lorsque la cible est en quatrième position.

\section{Le temps de sélection des références documentaires pertinentes}

Pour chaque condition, nous avons calculé l'intervalle de temps séparant l'affichage de la référence documentaire cible et la sélection de cette référence cible, ce qui correspond à l'action de "mettre dans le panier ». L'examen de ces temps de sélection nous permet d'estimer le temps nécessaire aux élèves pour juger comme adéquate une référence documentaire effectivement adéquate par rapport au thème d'une RDI selon la familiarité du thème, la position de la référence cible et 
l'adéquation des références qui la précédent. Les temps sont exprimés en dixièmes de seconde (figure 3 ).

Des analyses de variance multiples montrent que l'interaction entre le niveau de familiarité thématique, la position de la référence cible et l'adéquation des références précédentes est significative $\left(F_{(1-33)}=44,34, p<.01\right)$. Les résultats montrent également que le temps nécessaire à la sélection d'une référence documentaire cible augmente de manière significative lorsque cette référence cible est précédée de trois autres références documentaires $\left(F_{(1-33)}=49,51, p<.01\right)$, que celles-ci soient adéquates ou non (respectivement, $F_{(1-33)}=29,70, p<.01$ et $F_{(1-33)}=27,86, p<.01$ ) et que le thème soit familier ou non (respectivement $F_{(1-33)}=36,94, p<.01$ et $\left.F_{(1-33)}=13,23, p<.01\right)$.

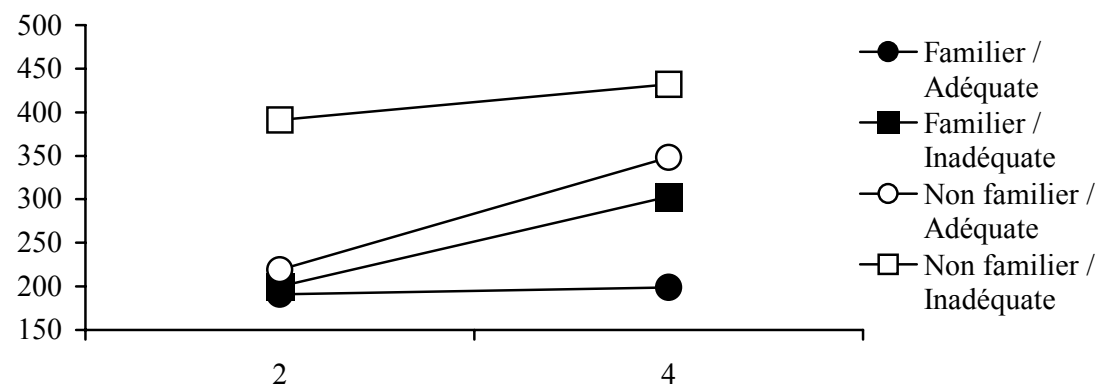

Position de la référence cible

Figure 3. Temps de sélection des références documentaires cibles (en dixièmes de seconde).

De plus, le temps nécessaire à la sélection d'une référence documentaire cible augmente significativement lorsque les références documentaires qui précèdent cette référence cible sont inadéquates $\left(F_{(1-33)}=101,87, p<.01\right)$, qu'il y en ait une ou trois (respectivement, $F_{(1-33)}=59,34, p<.01$ et $F_{(1-33)}=61,80, p<.01$ ) et que le thème soit familier ou non (respectivement, $F_{(1-33)}=46,37, p<.01$ et $F_{(1-33)}=82,66, p<.01$ ).

Enfin, les résultats des analyses de variance indiquent que le temps nécessaire à la sélection d'une référence documentaire cible augmente globalement lorsque le thème est peu familier $\left(F_{(1-33)}=108,26, p<.01\right)$. Cette augmentation est observée quelle que soit la position de la référence cible (en seconde position, $F_{(1-33)}=52,24$, $p<.01$ et en quatrième position, $\left.F_{(1-33)}=40,08, p<.01\right)$ et que les références qui précèdent la référence sonde soient adéquates ou non (respectivement, $F_{(1-33)}=30,37$, $p<.01$ et $\left.F_{(1-33)}=96,05, p<.01\right)$.

En d'autres termes, le temps pour sélectionner la référence documentaire cible augmente considérablement si cette référence cible est précédée de plusieurs autres références documentaires. De plus, cette augmentation est amplifiée lorsque les références documentaires qui précèdent la référence cible sont inadéquates par rapport au thème de la RDI. Enfin, l'interaction est d'autant plus importante que le thème de la RDI est peu familier pour les élèves. 


\section{CONCLUSIONS, IMPLICATIONS}

Bien que les élèves qui ont participé à notre expérience ne présentaient pas de difficultés majeures quant à leurs capacités de lecture-compréhension et de mémorisation, les résultats montrent que la familiarité du thème de la RDI, la longueur des listes de références documentaires et l'adéquation de ces références ont des influences sur le jugement de pertinence des références documentaires. Ainsi, la plupart de nos hypothèses sont confirmées. En effet, le nombre moyen de références documentaires cibles (qui sont toujours pertinentes) sélectionnées par les élèves diminue lorsque la liste de références augmente (hypothèse 1) et lorsque le thème leur est peu familier (hypothèse 3). L'effet de l'adéquation des références qui précèdent les cibles n'est constaté que lorsqu'elles sont non pertinentes. De plus, le temps nécessaire aux élèves pour sélectionner les références documentaires cibles, c'est-à-dire le temps nécessaire pour juger ces références comme effectivement pertinentes, augmente lorsque la liste de références augmente (hypothèse 4), lorsque le thème est peu familier (hypothèse 5) et lorsque les références qui précèdent les cibles sont inadéquates (hypothèse 6). Enfin, quel que soit l'indicateur comportemental (nombre de références cibles sélectionnées ou temps nécessaire à la sélection des cibles), les résultats montrent que les trois facteurs interagissent.

Les résultats tendent donc à valider l'hypothèse générale selon laquelle la cohorte conceptuelle activée après le traitement des consignes et du thème se dégrade au fur et à mesure du traitement de listes de références documentaires. De plus, notre expérience démontre que certains facteurs peuvent accélérer cette dégradation chez les jeunes élèves. Ainsi, le vecteur d'activation initial de la cohorte conceptuelle construit lors du traitement des consignes et du thème décline considérablement lorsqu'il y a de nombreux concepts « entrants » en mémoire suite au traitement de plusieurs références documentaires. Du fait des capacités limitées de la mémoire de travail, le nouveau vecteur d'activation provoque « l'effacement » $\mathrm{du}$ vecteur initial et donc l'oubli progressif des concepts pertinents par rapport au thème de la RDI. Cet " effacement» est d'autant plus rapide lorsque le nouveau vecteur d'activation ne comprend pas les mêmes concepts que ceux présents dans le vecteur initial, c'est-à-dire lorsque l'élève est amené à traiter beaucoup de matériel non pertinent par rapport au thème de la RDI. De plus, lorsque le thème de la RDI est peu familier pour les élèves, le vecteur initial est très limité puisque l'activation n'a pas pu, lors du traitement des consignes et du thème, se propager à de nombreux concepts en mémoire. Ce vecteur initial est donc «fragile» et encore plus facilement remplaçable par un nouveau vecteur consécutif au traitement de nouvelles références documentaires. Quoi qu'il en soit, le modèle élaboré par van den Broek et al. $(1995,1996,1999)$ semble être un outil approprié pour rendre compte des processus cognitifs sous-jacents à l'activité de sélection de références documentaires parmi des listes de références.

\section{Implications pédagogiques}

Pour certains, l'utilisation des nouvelles technologies dans les pratiques éducatives doit avoir pour principal objectif le développement de l'autonomie de l'élève dans la réalisation de certaines activités. D'ailleurs, pour les volontés institutionnelles, « l'élève doit, par son travail personnel ou en groupe, être capable 
de rechercher une information [et] il doit être capable d'utiliser l'ordinateur pour une recherche simple de documentation » (Les cycles de l'école primaire, 1991, p.36). Or, notre étude tend à montrer que, du fait de la dégradation progressive de la représentation mentale pertinente par rapport au thème de la RDI, les élèves de CM2 oublient progressivement les concepts constitutifs de cette représentation. De plus, cet oubli peut être accentué par certains facteurs, dont la familiarité thématique, la longueur de la liste de références à traiter et le degré de pertinence de ces références. Les résultats reportés ici montrent qu'un élève de CM2 peut éprouver de grandes difficultés à évaluer la pertinence de références documentaires, bien que cet élève ne présente pas de difficultés majeures quant à ses capacités de lecture-compréhension et de mémorisation. Ces difficultés correspondent à une baisse de la qualité du jugement de pertinence et à une hausse du temps nécessaire pour effectuer ce jugement. Il est raisonnable de penser qu'une aide extérieure, par exemple un rappel explicite des concepts initiaux pertinents, peut aider les élèves de CM2 à réaliser de manière efficace l'étape de sélection de références documentaires. Si la nécessité de se rappeler activement de l'objectif d'une RDI est intuitivement assez triviale, son explication psychologique n'est pas aussi directe qu'il n'y paraît. D'un point de vue pragmatique, le maintien de l'objectif exige une certaine connaissance et/ou l'expérience des tâches de RDI, et donc un apprentissage (Hirsh, 2000 ; Kuhlthau, 1999). Le sujet confronté à ces situations apprend progressivement à porter une attention particulière à l'instruction de traitement que constituent les consignes et le thème de recherche, parce que cette conduite est indispensable à " l'ajustement » de sa conduite (Allal \& Saada-Robert, 1992) et donc à la réussite de la tâche. Il s'agit d'un cas de conduite méta cognitive qui présuppose la connaissance de soi-même en tant qu'agent cognitif, et une certaine conscience des limites de la mémoire (Flavell, 1976, 1979). Or, ce type de connaissance réflexive se développe tardivement et très inégalement selon les domaines d'activités.

\section{Implications de conception}

Aujourd'hui, de nombreux outils de RDI et de recherche d'information peuvent être utilisés par les jeunes élèves. Afin de lutter contre ce que les spécialistes de la documentation nomment le «silence documentaire », l'un des objectifs des concepteurs de ces outils a été d'offrir le maximum de résultats pour une requête documentaire donnée. Ainsi, à force de lutter contre le «silence documentaire », est apparu le «bruit documentaire» qui correspond à une surabondance de résultats. Au vu de notre étude, cette volonté d'offrir un maximum de références à l'utilisateur semble contradictoire avec les capacités des jeunes élèves puisque la qualité du jugement de pertinence d'élèves de CM2 diminue lorsque augmente la quantité de matériel à traiter. Parallèlement à cette diminution, le temps nécessaire au jugement augmente. En d'autres termes, plus un élève de CM2 a de longues listes de matériel à traiter, c'est-à-dire de références documentaires à lire et à comprendre, plus le temps pour évaluer la pertinence de ces références augmente et moins cette évaluation est correcte. Il semble donc inutile voire «néfaste» du point de vue cognitif d'offrir de nombreuses références documentaires à des jeunes élèves en réponse à une requête documentaire.

De plus, l'accroissement massif du nombre de résultats obtenus pour une requête documentaire donnée s'accompagne généralement d'une hétérogénéité quant 
à la pertinence de ces réponses. En effet, parmi les nombreux résultats proposés par certains outils de RDI, beaucoup peuvent se révéler peu pertinents. Or, notre expérience tend à montrer que le fait de devoir traiter plusieurs références documentaires inadéquates par rapport au thème de la RDI contribue à l'oubli progressif de ce thème chez des élèves de CM2. Afin d'offrir d'une part un nombre limité de références documentaires et d'autre part des références qui sont adéquates, certains concepteurs élaborent des algorithmes pour calculer le degré de pertinence des réponses proposées par l'outil. À titre d'exemple, dans la nouvelle version de BCDI 2 école (BCDI 3 école, CRDP de Poitou-Charentes), les références documentaires seront proposées à l'élève par ordre décroissant de pertinence. Mais, la construction de ces algorithmes est loin d'être aisée du fait de la difficulté à définir la notion de « pertinence » et du fait des contraintes liées aux systèmes.

\section{BIBLIOGRAPHIE}

Allal L. \& Saada-Robert M. (1992). «La métacognition : cadre conceptuel pour l'étude des régulations en situation scolaire », Archives de psychologie, vol. 60, p. 265-296.

Barès M. (1984). La recherche documentaire dans le contexte télématique: modalités d'automatisation et utilisation des bases de données, Paris : Hachette.

Borgman C. L., Gallagher A. L., Kriegger D. \& Bower J. (1990). " Children's use of an interactive catalog of science materials ", in D. Henderson (éd.), Proceedings of the 53rd American Society for Information Science Annual Meeting, vol. 27, November 4-8, 1990, Totonto : Learned information, p. 55-68.

Brorowczak M. \& Machut J.-F. (1997). Pertinence de choix d'un document, IUFM de Lille $1:$ mémoire non publié.

Case R., Kurland M. D. \& Goldberg J. (1982). «Operational efficiency and the growth of short-term memory span », Journal of Experimental Child Psychology, vol. 33, p. $386-404$.

Chollet K. (1997). Les stratégies cognitives nécessaires à la recherche d'informations chez des enfants âgés de 9 à 13 ans, Poitiers, département de psychologie : mémoire de maitrise non publié.

Dinet J., Rouet J.-F. \& Passerault J.-M. (1998). «Les "nouveaux outils" de recherche documentaire sont-ils compatibles avec les stratégies cognitives des élèves ? », in J.-F. Rouet \& B. de La Passardière (éds), Hypermédias et Apprentissages 4, Actes du Quatrième Colloque Hypermédias et Apprentissages, Poitiers, 15-17 octobre 1998, Paris : INRP/EPI, p. 149-162.

Flavell J. H. (1976). « Metacognitive aspects of problem-solving », in L. B. Resnik (éd.), The nature of intelligence, Hillsdale, NJ : Lawrence Erlbaum Associates, p. 231-235.

Flavell J. H. (1979). « Metacognition and cognitive monitoring: A new area of cognitive developmental inquiry », American Psychologist, vol. 34, p. 906-911.

Hirsh S. G. (2000). « Children's relevance criteria and information seeking on electronic resources ", Journal of the American Society for Information Science, vol. 50, n ${ }^{\circ} 14$, p. $1265-1283$.

Kintsch W. \& van Dijk T. A. (1978). "Toward a model of text comprehension and production », Psychological Review, vol. 85, n 5, p. 363-394. 
Kintsch W. (1998). Comprehension: A paradigm for cognition, Cambridge : Cambridge University Press.

Kuhlthau C. C. (1991). «Inside the search process: Information seeking from the user's perspective », Journal of the American Society for information Science, vol. 42, p. 361-371.

Kuhlthau C. C. (1999). " The role of experience in the information search process of an early career information worker: Perceptions of uncertainty, complexity, construction, and sources ", Journal of the American Society for information Science, vol. 50, $\mathrm{n}^{\circ} 5$, p. $399-412$.

Lefavrais P. (1989). LIREL : La pipe et le rat, Paris : EAP.

Lefort G. (1990). Savoir se documenter, Paris : Les Éditions d'Organisation.

Les cycles à l'école primaire (1991). Programmes officiels du Ministère de l'Éducation nationale, Paris : CNDP.

Marchionini G. (1995). Information seeking in electronic environments, Cambridge : Cambridge university Press.

Markey K. (1987). « Searching and browsing the Dewey decimal classification in an online catalog », Cataloging and Classification Quaterly, vol. 7, p. 37-68.

Mizzaro S. (1998). «Relevance: The whole history », in T. B. Hahn \& M. Buckland (éds), Historical studies in Information Science, Medford, NJ : Information Today, p. 221244.

Moore P. A. \& St George A. (1991). «Children as information seekers: The cognitive demands of books and library systems », School Library Media Quaterly, vol. 19, p. 161-168.

Osmont B. (1995). Dynamiques cognitives et stratégies d'utilisateurs, Paris : Masson.

Rouet J.-F. \& Tricot A. (éds) (1998). « Chercher de l'information dans un hypertexte : vers un modèle des processus cognitifs ", in Les hypermédias, approches cognitives et ergonomiques, Paris : Hermès, p. 57-74.

Siegel L. S. \& Ryan E. B. (1989). «The development of working memory in normally achieving and subtypes of learning disabled children », Child Development, vol. 60, p. 973-980.

Solomon P. (1993). "Children's information retrieval behavior: A case analysis of an OPAC », Journal of the American Society for information Science, vol. 44, p. 222244.

Sutter E. (1994). «Les logiciels documentaires », in A. Vuillemin (éd.), Les Centres de Documentation et les Nouvelles Technologies de l'Information, Paris: La Documentation Française, p. 341-349.

Van den Broek P., Fletcher C. R. \& Risden K. (1993). «Investigations of Inferential Processes in Reading: A Theoretical and Methodological Integration », Discourse Processes, vol. 16, p. 169-180.

Van den Broek P., Risden K. \& Husebye-Hartmann E. (1995). «The role of readers' standards for coherence in the generation of inferences during reading », in R. F. Lorch \& E. J. O'Brien (éds), Sources of coherence in reading, Hillsdale, NJ : Lawrence Erlbaum Associated, p. 353-373. 
Van den Broek P., Risden K., Fletcher C.R. \& Thurlow R. (1996). «A "Landscape view" of reading: fluctuating patterns of activation and the construction of a stable memory representation », in B. K. Britton \& A. G. Graesser (éds), Models of understanding text, Mahwah, NJ : Lawrence Erlbaum Associates, p. 165-187.

Van den Broek P., Young M., Tzeng Y. \& Linderholm T. (1999). « The landscape model of reading: inferences and the on-line construction of a memory representation ", in $\mathrm{H}$. van Oostendorp \& S. R. Goldman (éds), The construction of mental representation during reading, Mahwah, NJ : Lawrence Erlbaum Associates, p. 71-98. 